ISSN $0208-5658$

Praca wplynęla do Redakcji dnia 13 maja 1997r.
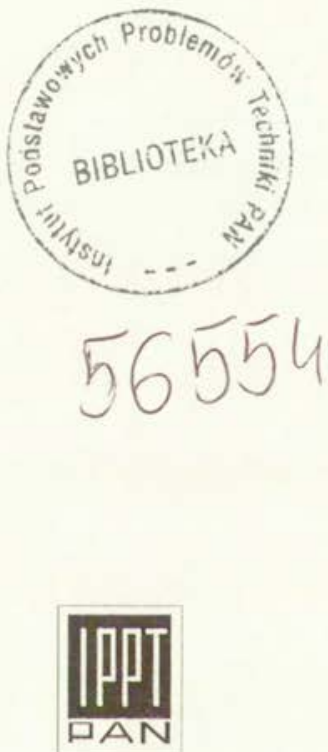

$\mathrm{N}$ a prawa $\mathrm{ch}$ ręk o p i s u

Instytut Podstawowych Problemów Techniki PAN

Nakład 100 egz. Ark. wyd. 1,0 Ark. druk. 1,5 Oddano do drukarni w maju 1997

ATOS Poligrafia-Reklama, Warszawa, Stawki 14 


\title{
EXPERIMENTAL VALIDATION OF NUMERICAL CODES IN THERMALLY DRIVEN FLOWS
}

\author{
Tomasz A. Kowalewski \\ Center of Mechanics, IPPT PAN \\ Polish Academy of Sciences, PL 00-049 Warszawa, Poland
}

\begin{abstract}
The focus of this review is application of modern full field experimental techniques based on the digital image analysis in venfyng and validating numerical solutions of thermally driven flows. Digital Particle Image Velocimetry and Thermometry, the new experimental methods based on a computational analysis of the colour and displacement of liquid crystal tracers, was used to obtain quantitative $2-D$ temperature and velocity fields information. The paths of the individual tracers obtained using 3-D digital particle tracking helped to understand and verify the flow structure. Laminar natural convection of liquids in small cube-shaped cavities, with and without phase change was studied experimentally and compared with numerical predictions. Implications arising from simplifications present in the numerical models are discussed.
\end{abstract}

\section{INTRODUCTION}

With the growing capacity of computers and continuing improvement in numerical codes, the question of the accuracy of numerical solutions is of primary importance. The usefulness of the numerical solution depends on its ability to model physical problem. Often the results following from idealized models, limited only to global description in terms of non-dimensional flow and heat flux characteristics, are accepted and successfully applied in engineering applications. However, there is a wide class of practical problems where knowledge of just the general behaviour of flow is not sufficient to obtain a full quantitative explanation of the phenomena. Examples include the distribution of fuel or soot in a combustion chamber, the transport of impurities in crystal growth, and the propagation of pollution in air and water. In such cases, the knowledge of some specific flow details is necessary for the full control of the investigated phenomenon. Improvement in the accuracy of theoretical and numerical models and their experimental validation is an indispensable procedure in such cases. With recent progress of experimental methods, introduced by digital image recording and analysis techniques, validation of numerical codes using full field experimental data became one of the most challenging research goals nowadays.

Obviously verification of a numerical scheme, grid refinement, and careful quantification of solution uncertainty are necessary steps towards the target. However, even the best tested code, positively verified against numerical „bench marks", solves the given system of PDE with some order of accuracy. The practical meaning of this accuracy depends strongly on the physical problem and the question posed. In that sense there is no universal measure of the physical accuracy of solution. Code verification, based on comparison of one solution values and their errors against another trusted in solution, only confirms (or not) whether the given set of equations was properly solved at the selected control points. In practice, $a$ priori error prediction of numerical modelling is seldom possible, and only a posteriori the confrontation of the solution with its physical counterpart remains. But not only the numerical uncertainty generates considerable problems in engineering applications. Another source of discrepancies are the PDE

$$
\text { http://rcin.org.pl }
$$


conceptual model assumptions which are necessary to describe the problem within numerical limits of time and space domains. Limited number of variables and equations used, simplifications of initial and boundary conditions and idealized fluid properties are intimate to any solvable numerical or analytical model of the physical problem. Whether or not those solutions bear any relation to a physical problem of interest is the subject of model validation. On the other hand, quite often numerical codes, both in engineering and research aspects, are used for obvious reasons with a low number of mesh points, far from ensuring mesh independence. Comparison of the output of these computations with experimental data will be able to show most of the essential physics of the flow, but generally without finer details. Validation at this level is rather considered as "calibration" of the numerical model against the experimental data. Without prior model validation the calibration procedure seems meaningless. But without the code calibration the model validation can become difficult if possible at all. Far more difficult to "calibrate", nevertheless very important, is the dependence of the numerical output on geometry, type of the computational mesh, the numerical method used and its order. Hence, we rather tend to extend the semantic defined by Roache', and expect the experimental validation to answer a practical inquiry: Do both the conceptual model simplifications and their numerical approximations offer acceptable description of our physical problem? The acceptable answer means the result which matches the measurements within an experimental accuracy. Small differences between the measured and predicted values are difficult to separate from inevitable experimental errors. Therefore, failure of the validation procedure emerges rather as an evident quantitative or qualitative disagreement of the observed and calculated flow details intrinsic for the investigated phenomena. An actual example would be consideration of the $g-j i t t e r$, definitely negligible small effect for "standard" problems. An experimental validation procedure can easily demonstrate its importance in the space environment.

For thermally driven flow the velocity and temperature fields are primary quantities. In the past, the pcint measurements were preferred to validate numencal codes. Despite the high accuracy characterizing the point methods (e.g. Laser Doppler Anemometry), a limited number of simultaneously acquired values make their use for the validation procedure potentially questionable. Therefore, we firmly believe that $2-D$ or 3-D flow field acquisition methods are the only alternative, especially for transient phenomena. With this objective in view, new experimental techniques were developed by the author and his co-workers and applied to the study of heat and mass transfer problems in flow of liquids. The primary method is based on a computational analysis of the colour and displacement of liquid crystal tracers, and it is used to determine both the temperature and velocity fields of the flow. It combines Digital Particle Image Thermometry (DPIT) and Digital Particle Image Velocimetry (DPIV). Full 2-D temperature and velocity fields are determined from a pair of colour images taken for the selected cross-section of the flow. Furthermore, a 3-D flow structure can be reconstructed from a few sequential measurements, if the flow relaxation time is sufficiently long. In some cases even apparently good agreement of the measured and calculated velocity and temperature fields does not guarantee their equivalence. The residual discrepancies may result in evidently different flow patterns. Detection of these differences in not an easy task. For this purpose, the second experimental method was introduced: automatic 3-D tracking of single tracer particles suspended in the fiow medium. Due to the strong sensitivity of the particle position to small additional forces or numerical inaccuracy, it could be demonstrated that the observed and simulated trajectories are often far from being in acceptable agreement, even for well known standard problems. In several cases also experimentally simpler $2-D$ particle tracking was found to be useful to demonstrate basic differences between the observed and calculated flow patterns.

Without approaching "fuzzy" territory of turbulence modelling, we try to elucidate the problem of experimental validation limiting our interest to the easier verifiable laminar fiow regime. Several analytical and numerical methods have been applied successfully to simulate and analyze this flow. However, despite all the research activities, very often details of the flow pattern cannot be predicted a priori from the given geometry and boundary conditions. The difficulty is primarily associated with the effects existing at the

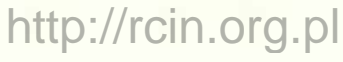


thermal and the velocity boundary layers because of the intimate coupling between boundary layer and core flows. The core flow is not readily determined from the boundary conditions but depends on the boundary layer which, in turn, is influenced by the core. This matter is not only of pure analytical nature but is of equal significance for numerical and experimental studies. Different scaling of the problems in the two regions is one of the sources of the errors or discrepancies generated by numerical codes. Another source of observed distinctions is a difference of several orders of magnitude in the velocity scale in one of the flow directions, complicating the three-dimensional modelling. In addition, thermal boundary conditions at non-isothermal walls are in practice neither perfectly adiabatic, nor perfectly heat conducting, as it is usually assumed in numerical models. Moreover, the widely used Boussinesq approximation for the physical properties of the fluid is not strictly valid for real fluids. A non-dimensional description of variable property fluid motion becomes difficult, if at all possible. The lack of such a description is an additional difficulty in the path of reconciling a given real flow and its different numerical models.

Our attempts to understand and explain the observed discrepancies between the measured and calculated convective flow patterns are reviewed for natural convection with and without phase change (freezing of water). To compare the experimental results, numerical simulations of transient and steady states were performed using mainly numerical codes developed by the CFD group at New South Wales University (G. de Vahl Davis, E. Leonardi and G. Yeoh).

\section{EXPERIMENTAL}

The experiments reported here concern the natural convection in small boxes filled with liquid. Both horizontally and vertically imposed temperature gradients were applied. In spite of the fact that the experiments were performed by author and his colleagues over several years and at different places (Max-Planck-Institut at Gottingen and IPPT PAN in Warsaw), main details of the experimental setup were preserved, modernizing acquisition methods only with time

The first investigated configuration (Figure la) concems a popular "bench mark" case, low Rayleigh number natural convection in a cubical cavity with differentially heated end walls ${ }^{34}$. Two opposite vertical walls are isothermal and kept at temperatures $T_{h}$ and $T_{c}$, the other four walls are nominally insulators of finite thermal diffusivity. A heat flux, both through and along the walls, is generated due to temperature gradients existing between the fluid inside the cavity and the surrounding environment and also along the front and back walls, the lid and the floor of the box. In the second configuration (Figure 1b), the top wall of the cube is isothermal at low temperature $T_{0}$. The other five walls are non-adiabatic, allowing a heat flux from external fluid surrounding the box. The temperature $T_{h}$ of the external bath is kept constant. Due to forced convection in the bath it can be assumed that the temperature at the external surfaces of the box is close to the bath temperature. The temperature field at the inner surfaces of the walls adjusts itself depending on both the flow inside the box and the heat flux through and along the walls. Both configuration were selected to investigate the convective flow with and without a phase change (freezing of water at the cold wall). To some extent they resemble a directional solidification in a Bridgman furnace used for crystal growth.

\section{Experimental set-up}

A typical experimental set-up used to acquire temperature and velocity fields consists of the convection box, a xenon flash or halogen tube lamp, and a CCD colour camera. Most of the experiments described here were performed using the cube-shaped cavity of $38 \mathrm{~mm}$ inner dimension. Either the two vertical opposite walls, or the top wall were made of a black anodized metal to maintain isothermal boundary conditions. The remaining walls were made of Plexiglas or glass. The temperature of the isothermal walls was controlled by thermostats. As a flow medium, pure

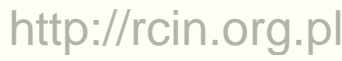


glycerine, its aqueous solutions and pure water were used. By varying the liquid composition and the temperature difference $\Delta T=T_{b}-T_{6}$, it was possible to cover a relatively wide range of Rayleigh and Prandtl numbers $\left(\operatorname{Ra}=2.10^{4}-3.10^{6}, \mathrm{Pr}=7-6900\right)$. The flow was observed at the vertical and horizontal cross sections of the cavity using a light sheet technique. The illumination system generated a $2 \mathrm{~mm}$ thick sheet of white light cutting the selected cross-section of the flow. A three-chip CCD colour camera, which gives an $R G B$-signal for the red, green and blue components of the incoming light, was used to observe the flow. The 24-bit images were acquired by frame grabbers (Eltec PPI or ITI PCI-CLR). Recording of the transient flow patterns and temperature fields was performed periodically. Typically every 10-300s short series of images were acquired and stored on the hard disk of the computer for later evaluation. The computer-controlled system of three step-motors combined with a mirror allowed to acquire images of several cross-sections, fully automatically within several seconds. Hence, due to relatively slow variations of the flow structures transient recording of main three-dimensional flow features was possible.

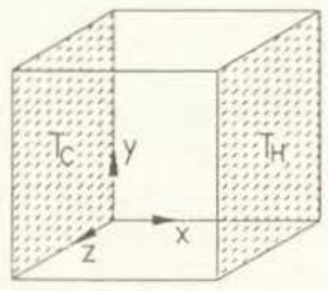

a

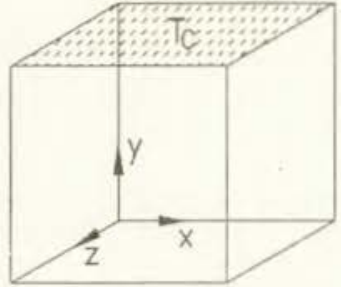

b

Figure 1. The cubical box with differentially heated walls (a) and cooled lid (b)

\section{Simultaneous temperature and velocity measurements}

The thermochromic liquid crystals (TLC) suspended in a working fluid were used both as tracers for the velocity evaluation and as local temperature sensors. The mean diameter of the $T L C$ particles was about $50 \mu \mathrm{m}$. The visualization of temperature using $T L C$ is based on their temperature-dependent reflectivity at the visible light wavelengths. If the liquid crystals are illuminated with white light, then the colour of the light they reflect changes from red to blue when the temperature is raised. This occurs within a well defined temperature range (the so-called colour play range), which depends on the type of TLC used.

For evaluating the temperature the $H S I$ representation ${ }^{5,6}$ of the $R G B$ space was used. The incoming $R G B$ signals were transformed pixel by pixel into Hue, Saturation and Intensity. Temperature was determined by relating the hue to a temperature calibration function. The non-linear relation between temperature and hue was described by a polynomial fitted to the measured points. The accuracy of the measured temperature depends on the hue value, and varies from $3 \%$ to $10 \%$ of the full colour play range.

The 2-D velocity vector distribution was measured by particle image velocimetry $(P I V)$. By this method, the motion of the scattering particles observed in the plane of the illuminating light sheet can be anaiyzed. The method applied here uses two separately captured digital images taken at a constant time interval (typically 1-5s) to evaluate the motion of the particles. Each of the images

$$
\text { http://rcin.org.pl }
$$


shows a relatively dense cloud of single illuminated particles (TLC). The magnitude and direction of the velocity vectors were determined using a cross-correlation analysis. This was done by dividing the whole image (typically $768 \times 512$ pixels) into small regularly spaced interrogation windows. The FFT-based correlation of the corresponding windows of both images enabled the evaluation of the mean translation vector for each group of particles simultaneously detected in both windows.

\section{Particle tracks}

To obtain a general view of the flow pattern, several images recorded periodically within a given time interval were added in the computer memory. Displayed images are similar to the multiexposed photographs, showing the flow direction and its structure. However, only a limited number of the two-dimensional projections of the particle tracks can be acquired this way. Better understanding of three-dimensional flow structures was possible using stereoscopic observation of particle tracks. For this purpose, a special procedure was developed ${ }^{8}$, which allows the detection and full automatic tracking of particles suspended in the flow medium. The images were captured by two CCD cameras (Sony XC77CE) integrated with two identical frame grabbers (VFG Imaging Inc.) installed in a personal computer, A few, nearly neutrally buoyant tracers of a diameter of about $150 \mathrm{~km}$ were observed against a bright background from two perpendicular directions. On-line identification of particles followed by the automatic data evaluation and storage enabled to earn unattended experiments over periods as long as several days 9.10

\section{NUMERICAL}

In conjunction with the experimental program, a numerical simulation of the problem was performed using a three-dimensional finite difference approximation of the Navier-Stokes and energy equations. Three numerical codes have been used in our investigations: TRFLOW ${ }^{11}$ which is a relatively flexible, vorticity - velocity code allowing simulation of transient convection of a constant property fluid; FRECON3D ${ }^{12}$ - a vorticity - vector potential false transient solver, superior for all studies of steady convection; and the most complex code FREEZE3D ${ }^{13,14}$ developed to study convection with phase changes. Owing the boundary-fitted grid generation, FREEZE3D allows to obtain precise front tracking solutions in the moving physical domain.

The computational models were adopted to simulate as closely as possible the physical experiment. When simulating experimental conditions, the main problem which arises is a proper definition of thermal boundary conditions $(T B C)$. In our study, either two opposite vertical walls or the horizontal top wall were assumed to be isothermal. The boundary conditions on the remaining walls, which are strictly neither adiabatic nor isothermal, were estimated by using the heat transfer theory applied to a thick, infinitely wide plane plate of uniform conductivity into an external unlimited environment. In this one-dimensional $T B C$ approach, an arbitrary temperature, a specific heat flux or a specific heat transfer coefficient on each of the six surfaces of the box was imposed in the calculations. Furthermore, in the modified versions of two last codes, the coupled 3-D solid-fluid heat conduction problem including bounding walls was solved together with momentum equation to improve modelling of the physical experiment. The effects of non-Boussinesq conditions (e.g. variation of fluid properties with temperature) on the resulting simulation were investigated. When variable properties are considered, the nondimensional description of the problem becomes only formal. Hence, dimensional physical data were used to specify the problem appropriately. For problem description, the non-dimensional parameters defined at the reference temperature were retained.

To check the validity of the numerical solution in comparison with the experimental results, several methods of numerical visualization were applied. In the first step the general flow characteristics, like

$$
\text { http://rcin.org.pl }
$$


two-dimensional temperature and velocity fields were extracted. A detailed visualization of the calculated flow structures was achieved with the help of particle tracks obtained through the integration of the velocity equations. To come closer to the physical experiment, the effects of additional forces acting on the real tracer particles have been studied ${ }^{10}$. The validity of the tracks has been verified in a number of ways, including reduction of the time step and a reversal of all velocities to enable the track to be traversed in reverse. Typical solutions were computed on a $31^{3}$ grid points (with 10 additional grid points in solid phase, where appropriate), chosen as a compromise between accuracy and cost. It has been found that this mesh is more than adequate if a global description of the flow is of interest. However, the localization of singularities and the determination of fine, threedimensional characteristics of the tracks such as the pitch of spirals appeared to be more sensitive to the mesh size, especially at higher values of the Rayleigh number.

\section{VALIDATION PROBLEMS}

Below we give few examples of the code validation problems which appeared during our investigations. They cover only a small fraction of the heat transfer problems, and the conclusions gained are not comprehensive. Our aim is to indicate rather where the problems may arise. In the description given below the observed effects are ordered into five groups. However, it is obvious that in the real flow any combination of them is possible.

\section{Thermal boundary conditions}

The general approach to the $T B C$ imposed on the limiting boundaries of a computational domain is very pragmatic. Due to usual lack of detailed information, idealizations like adiabatic, isothermal or imposed heat flux are widely acceptable. For sure, it is not efficient to extend the solution domain and calculate effects of the surrounding environment on the investigated flow. And usually it would be a superfluous complication. Whereas, most of the heat transfer problems do not suffer from $T B C$ model simplifications, it seems worth to sum up some of the consequences.

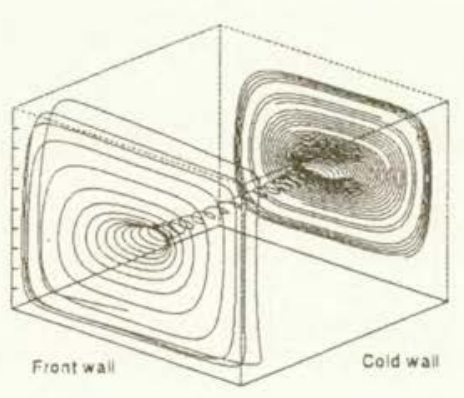

a

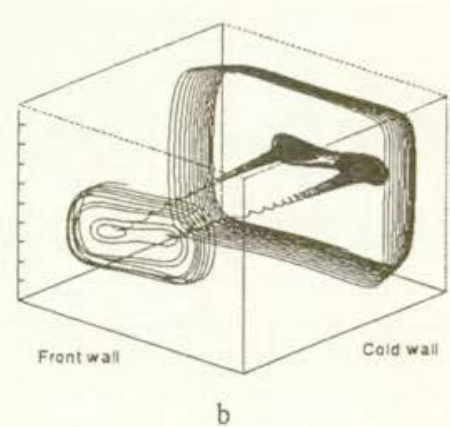

b

Figure 2. Calculated 3-D streamlines (front half of the cavity displayed only). $\mathrm{Pr}=6300$, adiabatic $T B C$; (a) - $\mathrm{Ra}=210^{4}$, inner spiral runs towards the centre; (b)- $\mathrm{Ra}=7.9 \cdot 10^{4}$, two inner spirals run towards the front wall. 
Our general observation coming from the investigated flow systems is that $T B C$ imposed at the nonisothermal walls effectively modify the three-dimensional flow structure. The main flow pattern usually remains well predictable for imposed temperature gradient, and can be described using simple 2-D modelling of the symmetry plane. However, the 3-D modelling becomes sensitive to $T B C$, when the flow out of the symmetry plane is analyzed. A good example of $T B C$ effects gives the standard "double glazing" problem investigated in the cube-shaped cavity. The flow pattern observed at the symmetry plane describes one or two vortex-like rings, transporting fluid back and forth between isothermal walls. This flow, being decisive for the overall heat transfer, is well predictable and rather insensitive to $T B C$ at the „side" walls. The 3-D flow structure consists in one or two spiralling motions, responsible, beside the main recirculation, for ,cross-flow” from the front and back walls to the cavity centre (Figure 2).

The numerical simulation using simple adiabatic $T B C$ for the side walls, has generally shown good agreement with the velocity and temperature fields measured at the symmetry plane Only small deviations in the isotherms appeared close to the top and bottom walls, due to the final heat conduction within the side walls. Investigations of the onset of convection studied for a similar case ${ }^{15}$ illustrate benefits of using $T L C$ tracers for instantaneous measurements of $2-D$ temperature and velocity fields. A sequence of images of the flow gives us a direct view of how, after applying a temperature jump at the hot wall, primary vertical isotherms continuously transform their shape into the final characteristic S-form (Figure 3). Also this transient behaviour remains in a good agreement with the $2-D$ or 3-D numerical counterparts obtained by assuming adiabatic $T B C$
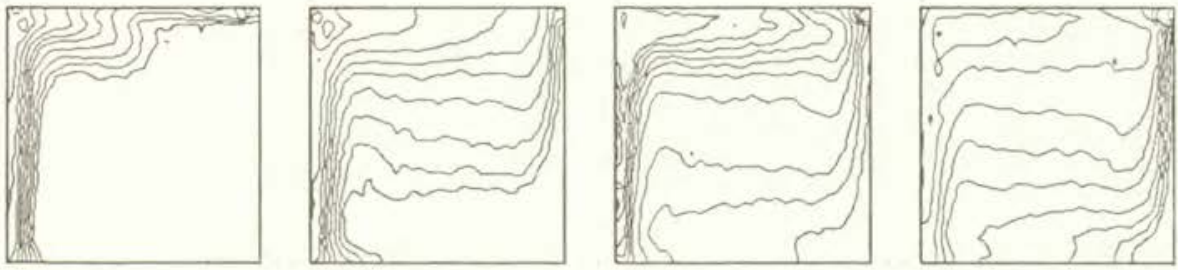

Figure 3. Transient development of isotherms measured at the centre-plane of differentially heated cavity: time $t=2 \mathrm{~min}, 10 \mathrm{~min}, 20 \mathrm{~min}$ and $60 \mathrm{~min}$ after the temperature $\mathrm{T}_{\mathrm{h}}$ is applied; initial fluid temperature $\mathrm{T}_{0}=\mathrm{T}_{\mathrm{c}}, \mathrm{R}_{\mathrm{a}}=1.810^{5}, \mathrm{P}_{r}=980$

These break down when approaching the front or back walls (the nominally adiabatic vertical walls) of the cavity. It was found ${ }^{4}$ that both the experimental isotherms and the flow structure differ from those simulated. Due to the physically non-adiabatic conditions on the side walls, the temperature field at those walls is characterized by larger horizontal gradients. For single roll system (low Rayleigh number) its axis becomes shifted towards one of the isothermal walls. The straight inner spiral resulting for adiabatic $T B C$ (Figure 2a), has in reality its ends curved towards the hot wall. For the two-roll system, only one spiral initially reaches the front and back wall. Two-roll flow structure forming characteristic „cats eyes" in the symmetry plane, apparently merge midway between the centre and side walls. Several numerical investigations have been performed to explore this effect. It appeared that the shape, location of the merging region, direction and pitch of the inner spirals are extremely sensitive to $T B C$ at all non-isothermal walls. Due to this sensitivity, the estimation of the proper $T B C$ for the given experiment becomes a non-trival task, especially for the two-roll system 


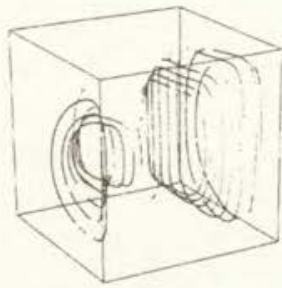

a

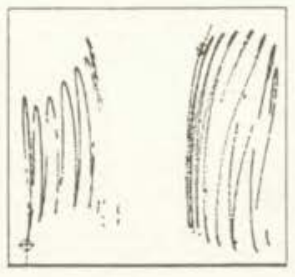

b

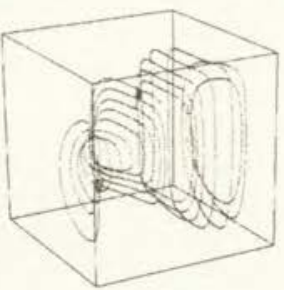

C

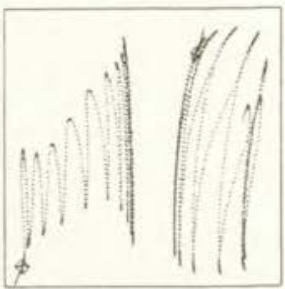

d

Figure 4. Particle tracks: a, b-measured, c,d-calculated using $T B C$ from the experiment; $R a=410^{4}$, $\mathrm{Pr}=1180$. Isometric view of the front half of the cavity and top view.

The trial and error method first used to fit the $T B C$ was replaced by a process of defining an explicitly measured temperature distribution for all four non-isothermal walls. Figure 4 shows a comparison between observed particle tracks and streamlines calculated with the experimentally defined wall temperatures. Both the direction of the calculated spirals and their pitch correlate well with the measured particle tracks. The improvement obtained gave us an indication of the necessity of code modifications, i.e, the $3-D$ modelling of heat transport through and along non-isothermal walls.
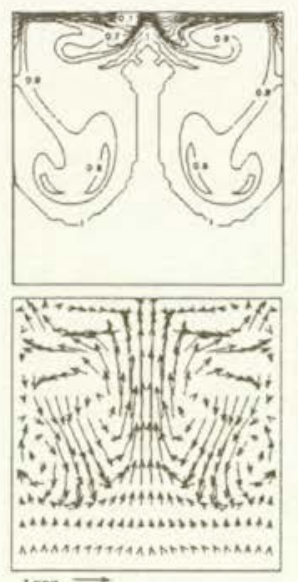

a
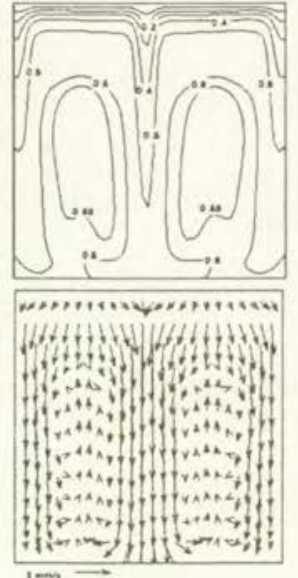

b
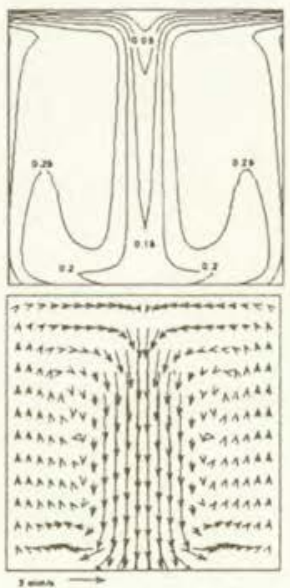

c
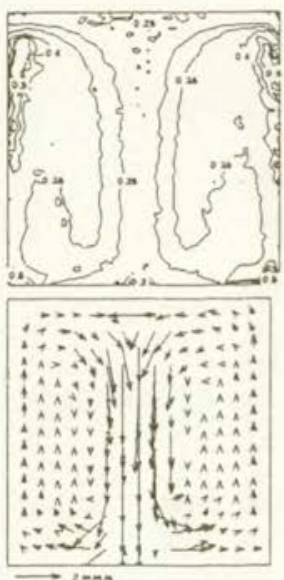

d

Figure 5. Onset of convection in the lid cooled cavity. $\mathrm{Ra}=2.710^{6}, \mathrm{Pr}=7$. Non-dimensional temperature (upper row) and velocity (lower row) at the centre symmetry plane. Numerical simulation for $40 \mathrm{~s}$ - (a), 200s - (b) and 60min -(c). Experimental data at $60 \mathrm{~min}$ - (d).

The onset of convection and the stability of an initially isothermal fluid in a cubical box instantaneously cooled from above have been extensively investigated for water, both with and without phase change. Physically this configuration bears some similarity to the Rayleigh-Benard 
problem. However, due to altered thermal boundary conditions at the side walls, the flow structure is different. The cubic symmetry of the box imposes a strong downward flow along the vertical axis of symmetry. However, before a stable final flow structure is achieved, several dramatic changes in its pattern are observed. The flow starts from the lid, when an initial cold thermal boundary layer becomes unstable, breaking down to four symmetrical plumes falling down along the side walls (Figure 5a). This in turn generates several recirculating zones, transporting heat and vorticity from the side walls to the centre. Within 3-5 minutes a general flow pattern is established. In the centre plane two ,jets" of cold liquid are streaming along the side walls, and another one along the vertical box axis (Figure 5b). This configuration is unstable. Depending on experimental disturbances or numerical noise present, after 4-8 minutes a dramatic flow pattern transformation takes place. Passing through several strongly asymmetrical flow forms, a final configuration with a single cold ,jet" along the cavity axis and a reverse flow along side walls are established (Figure $5 c$,d). The final state is reached after about 40 minutes.

The flow visualizations shown the existence of a complex spiralling structure transporting fluid up along the side walls and down in a central cold, jet" along the cavity axis. For walls of high heat conductivity (glass), eight symmetric cells were created by the flow. For Plexiglas walls additional small recirculation regions appeared separating the main cells. Although the computational results obtained for "idealized" I-D TBC confirmed the eight-fold symmetry of the temperature and flow fields observed experimentally, their orientation was different. Moreover, the measured isotherms were evidently shifted to higher values. Serious discrepancies were noticed for temperature distribution observed at the horizontal cross-section ${ }^{16}$

In several computational runs the heat flux was step-wise modified in the $1-D T B C$ model so as to give better agreement with the measured temperature profiles. It was found that such agreement could be obtained by assuming nearly twice as much heat flux through the side walls as the nominal value calculated from the physical characteristics of the side walls. It indicates that the heat flux along the side walls, neglected in the simple $1-D$ model of $T B C$, effectively increases the heat transfer from the external medium. The differences between the observed and calculated flow patterns remained even after such arbitrary modification of TBC. Consequently, the calculated streamlines starting in the diagonal symmetry plane spiralled in the opposite direction of that observed in experiments. Especially, for $8 \mathrm{~mm}$ thick Plexiglas walls, $T B C$ could not be properly modelled using a $I-D$ assumption about the heat flux. Physically it is possible for a flow pattern with an opposite sequence to develop, that is spiralling inwards in the central plane and outwards on the diagonal plane. Therefore only a slight change of the TBC may modify the flow pattern. This was observed by replacing the side walls of low conductivity Plexiglas by thin glass walls.

The numerical simulations performed for both cases using the $3-D$ model for wall heat conductivity confirmed the triggering mechanism of $T B C$ on the observed flow pattern. Inclusion of the side walls in the computational domain and solving the coupled fluid-solid heat conduction problem improved the agreement with the observed flow pattern. Also the observed temperature distribution as well as its symmetry were fully recovered in the numerical results ${ }^{17}$. It was only a result of use of both the experimental and numerical methods that the fine structures of the thermal flow were fully understood

The formation of ice was studied by decreasing the lid temperature to $-10^{\circ} \mathrm{C}$. A complicated flow pattern appeared after the convection started. This was also manifested in the complex structure of the ice surface Similar to the computed initial ice surface, a star-like grooving of the ice surface was observed in the experiment. It was found that the creation of the ice layer at the lid has a stabilizing effect on the flow. The grooving of the ice-fluid interface which formed on the lid of the cavity 
imposed the direction and character of the flow, damping the instabilities observed for the pure convection case. There is also a density inversion under the lid which decelerates the main ,jet" and limits the strong generation of vorticity in that region. The numerical results obtained for $1-D$ model of TBC exhibited only general agreement with the experiments. The predicted rate of ice growth was overestimated $^{18}$, especially for higher $T_{h}$. The $I-D$ modelling of heat transfer within side walls could be the responsible factor. Modification of the numerical code by solving the 3-D heat conduction problem within the thick Plexiglas walls appeared to be not sufficient (Figure 6). The additional influence of such effects like supercooling, inaccuracy of modeling the thermal boundary layer at the ice surface, non-homogeneous ice structure or heat conduction contact resistance at ice-wall surfaces, have to be investigated

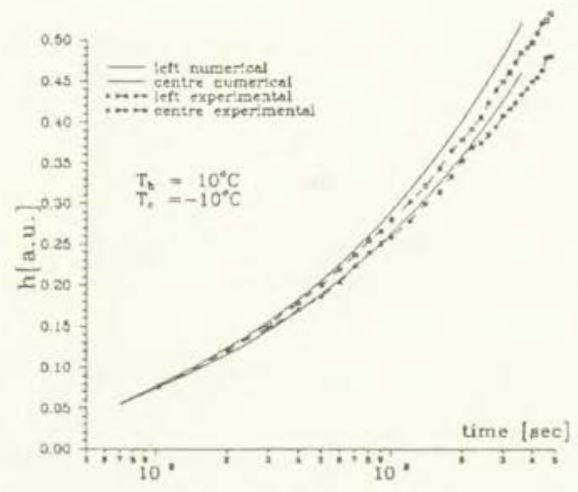

(a)

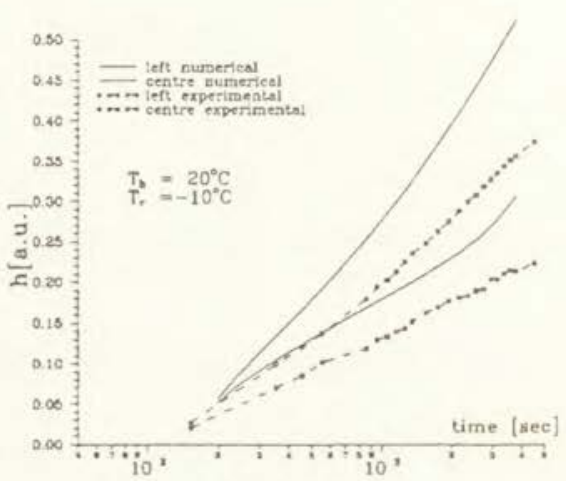

(b)

Figure 6. Measured (symbols) and calculated (solid lines) ice interface growth rate at the centre and at the side wall of lid-cooled cavity. Top wall at temperature $\mathrm{T}_{\mathrm{c}}=-10^{\circ} \mathrm{C}$, external temperature $\mathrm{T}_{\mathrm{h}}=10^{\circ} \mathrm{C}$ - (a) and $20^{\circ} \mathrm{C}-(\mathrm{b})$. Numerical simulation using 3-D TBC for $8 \mathrm{~mm}$ Plexiglas walls.

\section{Variable properties}

The so-called „variable properties" approach is understood as an antonym to the widely used Boussinesq approximation. The effects of non-Boussinesq conditions (e.g. variation of fluid properties with temperature) increases the problem complexity. It is because the functional relations of properties are specific to each fluid, more than one property of a single fluid may vary with temperature and the variation may differ within different temperature ranges. A non-dimensional description of variable properties fluid motion becomes difficult, if possible at all. The lack of such a description is an additional difficulty on the path of reconciling a given real flow and its different numerical models. Usually four properties have to be considered for liquids, the fluid viscosity, density, thermal conductivity and heat capacity. At small temperature variation, the changes of density and viscosity of typical liquids seems to be the most important. Even limiting our interest to these two functions, it is not possible to give a unique recipe, where and when these properties variation should be considered in the model. For example, the variation of viscosity seems to have significant impact on the bottom-heated configuration of a convective cell, the Rayleigh-Benard problem in bounded domains. Already in the early $30 \mathrm{~s}$ it was shown that the dependence of fluid viscosity on temperature may not only change the flow velocity profile but also has a qualitative 
triggering effect on the selection of the preferred direction of rotation of the Rayleigh-Bénard rolls ${ }^{19}$ Recent work of Khorasanizadeh ${ }^{x}$ gives an exhaustive study of the problem.

In our experiments with the horizontally heated cavity, the Rayleigh number was changed simply by increasing the temperature difference $\Delta \mathrm{T}$. This in turn increased the effect of the variable liquid properties. For glycerine as a fluid medium, the effect of temperature dependent viscosity produced up to $50 \%$ asymmetry in the velocity profiles $\left(\Delta \mathrm{T}=15^{\circ} \mathrm{C}\right)$. Comparison of temperature profiles indicated that the cold boundary layer became thicker due to the increased fluid viscosity A large portion of temperature drop (and the viscosity change) occurred in this layer. Hence, the convection motion becomes confined to the smaller region, the hotter part of the cell. The effects of viscosity variation are particularly striking by the onset of convection in the cavity initially filled with a cold fluid Observed penetration of the hot fluid generated by a sudden temperature gradient applied to the two opposite walls becomes apparently retarded by a stagnant layer close to the cold wall. For the free surface case, small recirculation regions in the lower part of the hot wall were observed, when the flow started from rest. Numerical simulation shows however, that effects of viscosity variation are secondary as compared with the $T B C$, if the behaviour of final state three-dimensional structures is of interest. Despite evident asymmetry of the velocity field, only small modification of the two-roll spiralling structure occurred.

The peculiarities in the physical behaviour of water have been the impetus for many studies of thermally driven flows in water at temperature near the maximum density point. In a numerical study of the effects of variable properties, Reizes et al. ${ }^{21}$ have shown for water that, in addition to highly non-linear density effects, also viscosity, thermal conductivity and specific heat effects can have a significant influence on the natural convection and heat transfer In our study of convection in the top-cooled cavity, the effects of density inversion of water followed by ice formation were investigated. For the lid temperature kept at $\mathrm{T}_{\mathrm{c}}=-10^{\circ} \mathrm{C}$ and external bath temperatures $\mathrm{T}_{\mathrm{h}}$ varying from $5^{\circ} \mathrm{C}$ to $25^{\circ} \mathrm{C}$, two horizontally stratified regions were observed. The first region, adjacent to the ice surface corresponds to the temperature range $0^{\circ} \mathrm{C}-+4^{\circ} \mathrm{C}$ In this regime, the buoyancy force acts everywhere upwards. Since the cooling surface is downward facing and horizontal, conditions of a stable stratification are present. In fact, for the external temperature of $5^{\circ} \mathrm{C}$ this region filled most of
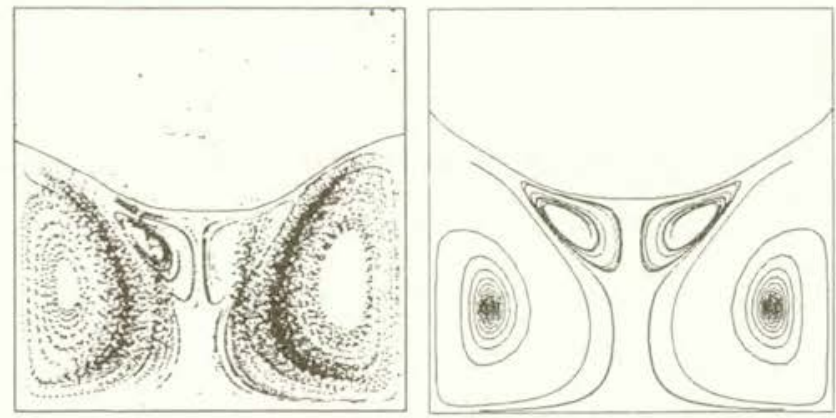

Figure 7. Natural convection underneath the ice surface. Particle tracks observed (left) and calculated (night) at the vertical centre plane. Lid-cooled Plexiglas cavity, $\mathrm{T}_{\mathrm{h}}=20^{\circ} \mathrm{C}, \mathrm{T}_{\mathrm{c}}=-10^{\circ} \mathrm{C}$ 
the cavity and the stable thermal stratification was observed. For higher external temperatures, the buoyancy driven convection flow developed in the second, bottom region. This in turn generated secondary motion in the adjacent top layer, intensifying heat transfer to the ice surface. Presence of the warm side walls additionally modified this configuration, allowing the hot liquid to penetrate the ice surface. Hence, initially flat surface of the ice crystal became conical. This modified the underneath flow, leading to the oscillatory motion observed when the flow starts. Large changes both of the flow pattern and the ice shape were observed when external temperature was changed. Moreover, the ,reverse" convection could be demonstrated, imposing the temperature $\mathrm{T}_{\mathrm{c}}=5^{\circ} \mathrm{C}$ at the lid and $\mathrm{T}_{\mathrm{h}}=0.5^{\circ} \mathrm{C}$ for the external bath.

A comparison of the measured and predicted ice/water interface and the associated flow structures can be seen in Figure 7. There is a primary cell which carries hot fluid up the wall to the ice interface, resulting in cutting back of the outer edges of this interface; than the water flows down in the centre of the cavity. Just below the ice in the centre of the cavity, there are small counter-rotating secondary flows established due to the density extreme of water in this region ${ }^{16}$.

\section{Supercooling}

Most of the investigations concerning solidification assume the isothermal conditions at the phase change boundary and the temperatures above the freezing point for the liquid phase. However, it is well known that usually the fluid supercooling precedes the phase change ${ }^{2}$. For example, water of standard purity will normally supercool to about $-5^{\circ} \mathrm{C}--7^{\circ} \mathrm{C}$ before the ice nucleation appears ${ }^{2}$. It may significantly retard the solidification process. Furthermore, freezing of the supercooled water results in the formation of the dendrite ice structure which becomes responsible for modification of the heat flux from the cooling wall. Thus a better understanding of the role of supercooling in the solidification process seems to be desirable. The proper modelling of the supercooling is not a trivial task. The supercooling depends on the purity of the fluid, concentration of the nucleation sites, the cooling rate or even the cooling history. Theoretical prediction of these parameters is very inaccurate. It is also not easy to extract quantitative information on the basis of empirical data only.

Our experiments with freezing water indicated that in most of the cases distilled water cools down to about $-7^{\circ} \mathrm{C}$ before phase change begins. In the lid-cooled cavity the supercooled layer of liquid remained attached to the cold wall. Its presence modifies the onset of convection. After about 60 s of cooling the lid, the first ice layer of about $1 \mathrm{~mm}$ thickness was observed. It started abruptly to propagate across the wall plane with horizontal growth rate of about $40 \mathrm{~mm} / \mathrm{s}$, several orders of magnitude faster than subsequent thickening of the ice layer. In the differentially heated cavity the observed effects of supercooling were even more dramatic, qualitatively changing the onset of freezing. In the cavity filled with warm water of $10^{\circ} \mathrm{C}$ sudden cooling of the side wall to $\mathrm{T}_{\mathrm{c}}=-10^{\circ} \mathrm{C}$ generated immediately the counter-clockwise vortex with upwards convection of the supercooled fluid. During the first 40-60s the supercooled water plum could cover one third of the upper surface, before its sudden freezing occurred. Then, within the next $10-15 \mathrm{~s}$, the clockwise convection melting the excessive ice at the lid recovered the "regular" plane propagation of the ice front. It seems obvious that the supercooling changes the initial circulation inside the cavity. The resulting heat transfer differs from that occurring in the "standard" situation. Also, due to the supercooling, the initial ice layer had usually a "milky" dendrite-like structure. Thermal conductivity of this non-homogeneous layer deviates from that of the pure solidus. Neither supercooling nor imperfection of the ice block was present in the numerical model. Perhaps this is one of the reasons of the difficulties we encountered in achieving agreement between numerical and experimental data. 


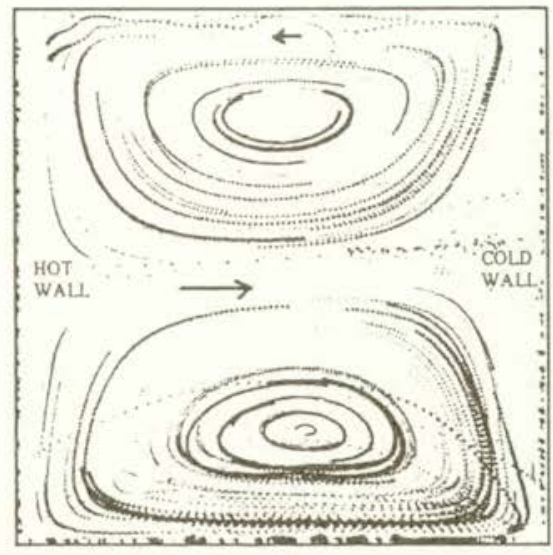

Figure 8. Flow pattern observed at the top surface of the fluid. $\mathrm{Ra}=395000, \Delta \mathrm{T}=5^{\circ} \mathrm{C}, 99$-foid exposure at 20 s intervals.

\section{Interfacial effects}

The free surface flow is a subject of vast literature, mainly in context of the low gravity conditions. Here, we would like to indicate that existence of surface effects may also modify the flow structure under „on earth" conditions. The series of free-surface experiments were performed for a differentially heated cube-shaped cavity filled with water-glycerine solutions ${ }^{24}$. As it was expected, the free surface evidently deformed regular flow structures increasing the hot-cold side asymmetry of the velocity field. Once the flow was fully developed, additional very slow but clearly visible recirculation vortex appeared directly at the surface. This flow seems to be de-coupled from the underneath ,regular" convection pattern. When the flow along the central part of the surface plane is directed towards the cold wall, reversal flow appears along the side walls (Figure 8).

In the numerical simulation of the problem, the standard velocity slip condition at the top boundary was introduced. Due to the strong buoyancy effects, the thermocapillary forces at the temperature difference applied $\left(\Delta \mathrm{T}=5^{\circ} \mathrm{C}\right)$ could be neglected. The overall agreement between the calculated and measured general flow characteristics appeared satisfactory. However, the observed recirculating flow at the surface was not found. Neither relatively strong effects of the variable fluid viscosity, nor non-adiabatic thermal boundary conditions could produce the observed recirculating flow at the surface. It seems that anisotropy of the molecular forces could be responsible for the observed stratification. Introduction of so-called surface viscosity may appear to be a necessary extension of the physical model.

In the solidification problem the interface forces at the solid-liquid interface may also play a significant role. However, its effect on the shape deformation is difficult to separate from other phenomena. At the moment it seems impossible to expose the effects of interface forces on the grooving observed at the ice surface. Perhaps precise measurements of the interface depletion could bring some evidence of an additional smoothing process. 


\section{Initial conditions}

An important element of a numerical model is its ability to describe correctly a true transient process. By modelling the transient process one needs to impose initial conditions, in our case the full fields description of the temperature and velocity fields. Proper definition of these conditions becomes sometimes doubtful, when comparison with experiments is necessary. In the experiment, for practical reasons it is not possible to achieve complete monitoring of the initial conditions. On the other hand, small deviation of the initial conditions may deform the transient flow behaviour or even generate different flow configurations. Hence, for the validation procedure the necessary step is to simplify the problem, imposing well controllable „standard" flow patterns.

In our experiments the following start-up situations were investigated: stagnant liquid initially at constant temperature (equal that of the hot wall, cold wall or mean temperature), or the fully developed initial convection pattern. The first situation seem to be well defined and well suited for the code validation. However, in practice these initial flow conditions are difficult to be controlled entirely. It is hard to keep less viscous fluid completely quiescent. Its residual motion is even necessary to maintain the uniform temperature. Hence, always some fluctuations of the temperature and velocity field are present. In the lid-cooled cavity these small initial fluctuations were observed to trigger instability of the thermal boundary layer, determining the whole transient flow scenario These led to serious difficulties in finding the proper numerical counterparts. Hence, several transient experiments were performed at modified initial conditions, allowing the fluid to develop well defined convection pattern before the sudden change of the temperature imposed at the isothermal walls triggered the analyzed transition. In this way, for the differentially heated cavity transition from the one to the two-roll system could be well described. For the lid-cooled cavity it allowed to achieve stable, symmetrical growth of ice and its quantitative comparison with the numerical model became possible.

\section{CONCLUDING REMARKS}

The method described of simultaneous measurement of the flow and temperature fields enables a relatively easy verification of global features of experimental and numerical simulations. Fine details of the flow can also be properly interpreted if the particle tracks are analyzed. The discrepancy between the predicted and observed flow patterns can be minimized if an interactive trail and error procedure is used, modifying „weak" points in the $T B C$, implementing the measured temperature fields as $T B C$, and improving the numerical code. In many engineering problems such a tedious procedure may seem to be unpractical. Unfortunately, at the moment we can not offer any universal "golden" rule, which could replace it. However, it has been found that a large improvement in quality and reliability of numerical simulation can be obtained by means of validations and tuning methodologies using information obtained from the flow visualization and full field measurements. 


\section{ACKNOWLEDGMENTS}

I gladly acknowledge the contribution of W. Hiller, G. de Vahl Davis, E. Leonardi, G. Yeoh, M. Behnia, F. Stella, St. Koch, C. Abegg, P. Mitgau, C. Soller, A. Cybulski and M. Rebow, to what has been a team effort over a number of years.

This work was supported by the Polish Scientific Committee (KBN Grant No. 3P40400107). Part of the computations were performed on CRAY-CS6400 at the Warsaw University of Technology (COI)

\section{REFERENCES}

1 Roache, P J., Quantification of uncertainty in computational fluid dynamics, Anmu. Rev. Fluid Mech., Vol. 29, pp. 123-160, 1997

2. Hiller, W, Kowalewski, T.A., Simultaneous measurement of the temperature and velocity fields in thermal convective flows, Flow Visualization IV, (Editor Claude Veret), pp. 617-622, Hemisphere, Paris 1987.

3. Hiller, W J., Koch, St, Kowalewski, T.A., Three-dimensional structures in laminar natural convection in a cube enclosure, Exp. Therm. and Fluid Sci., Vol, 2, pp 34-44, 1989

4. Hiller, W J., Koch, St., Kowalewski, T.A., de Vahl Davis, G., Behnia, M, Experimental and numerical investigation of natural convection in a cube with two heated side walls, Proc. of IUTAM Symposium, Cambridge UK, Aug. 13-18, 1989, (Edits. H.K. Moffat \& A. Tsinober), pp 717 - 726, CUP 1990.

5. Koch, St, Beruhrungslose Messung von Temperatur und Geschwindigkeit in freier Konvektion, Ph.D. Thesis in Mitteilungen aus dem MPI 108, Gottingen, 1993

6. Hiller W. J., Koch, St., Kowalewski, T.A., Mitgau, P., Range, K., Visualization of 3-D natural convection, Proceedings of The Sixth International Symposium on Flow Visualization, Eds. Tanida Y. \& Miyashiro H., pp 674-678, Springer-Verlag. 1992.

7. Paul, R, Die Erfassung von Geschwindigkeitsfeldern durch automatische Bewegungsanalyse am Beispiel einer rotierenden Flüssigkeit, Ph.D. Thesis in Mitteilungen aus dem MPI 102, Göttingen, 1991.

8. Bartels-Lehnhoff, H.-H., Erfassung dreidimensionaler Bahnlinien und Geschwindigkeitsfelder mittels automatischer Bildverarbeitung, Ph.D. Thesis in Mitteilungen aus dem MPI 101, Göttingen, 1991

9 Mitgau P.M., Hiller, W.J., Kowalewski T.A., Verfolgung von Teilchen in einer dreidimensionalen Strömung, ZAMM, Vol. 74(5), pp T394-396, 1994.

10. Yarin, A., Kowalewski T.A., Hiller, W.J., Koch, St., Distribution of particles suspended in 3D laminar convection flow, Physics of Fluids, Vol. 8, pp. 1130-1140, 1996.

11. Guj, G., Stella, F, A vorticity-velocity method for the numerical solution of 3D incompressible flows, J. Comp. Physics, Vol. 106, pp 286-298, 1993. 
12. Goh, L.P., Leonardi, E. \& de Vahl Davis, G., FRECON3D -Users Manual. A program for the numerical solution of mixed convection in a three-dimensional rectangular cavity, Report 1988 FMT/7, University of New South Wales, Sydney 1988.

13. Yeoh, G.H., Natural convection in a solidifying liquid, Ph.D. Thesis, University of New South Wales, Sydney 1993

14. Yeoh, G.H., Behnia, M., de Vahl Davis, G., Leonardi E., A numerical study of three-dimensional natural convection during freezing of water, Int. J. Num. Meth. Eng., Vol. 30, 899-914, 1990.

15. Hiller, W. J., Koch, St., Kowalewski, T A. \& Stella, F., Onset of natural convection in a cube, Int. J. Heat Mass Transfer, Vol. 36, pp 3251-3263, 1993.

16. Abegg, C., de Vahl Davis, G., Hiller, W.J, Koch, St., Kowalewski, T.A., Leonardi, E., Yeoh, G.H., Experimental and numerical study of three-dimensional natural convection and freezing in water, Proc. of 10th Int. Heat Transfer Conf., Vol, 4, pp 1-6, Edt. G.F. Hewitt, IChemE 1994.

17. Soller C., Hiller W.J., Kowalewski T.A. \& Leonardi E., Experimental and numerical investigation of convection in lid cooled cavities - effects of non-ideal thermal boundary conditions on three-dimensional flow, paper presented at 3rd ICIAM Congress, Hamburg 1995.

18. Kowalewski, T. A. Cybulski A., Experimental and numerical investigations of natural convection in freezing water, Int. Conf. on Heat Transfer with Change of Phase, Kielce (Poland), Dec. 8-10, 1996, in Mechanics, Vol. 61/2, pp. 7-16, 1996.

19. Graham, A, Shear patterns in an unstable layer of air, Phil. Trans., Vol. A 296, pp 469-475, 1933

20. Khorasanizadeh, H., A numerical and experimental study of Rayleigh-Bénard convection, Ph.D. Thesis, UNSW Sydney 1997.

21. Reizes, J., Leonardi, E. \& de Vahl Davis, G., Natural convection near the density extremum of water, Proc. of Fourth Int. Conf. on Numerical Methods in Laminar and Turbulemt Flow, pp 794-804, U.K. 1985.

22. Knight, C.A., The freezing of supercooled liquids, D. Van Nostrand Co., Toronto 1967.

23. Brewster, R.A., Gebhart, B., An experimental study of natural convection effects on downward freezing of pure water, Int. J. Heat Mass Transfer, Vol. 31, pp 331-348, 1988.

24. Kowalewski, T.A., Cybulski, A., de Vahl Davis, G., Leonardi, E., Free surface natural convection in differentially heated rectangular cavity, paper presented at 19th ICTAM Congress, Kyoto, 1996

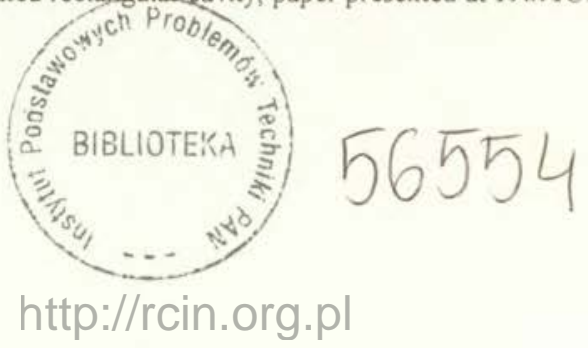


Colour plate

Visualization of the temperature field using liquid crystals

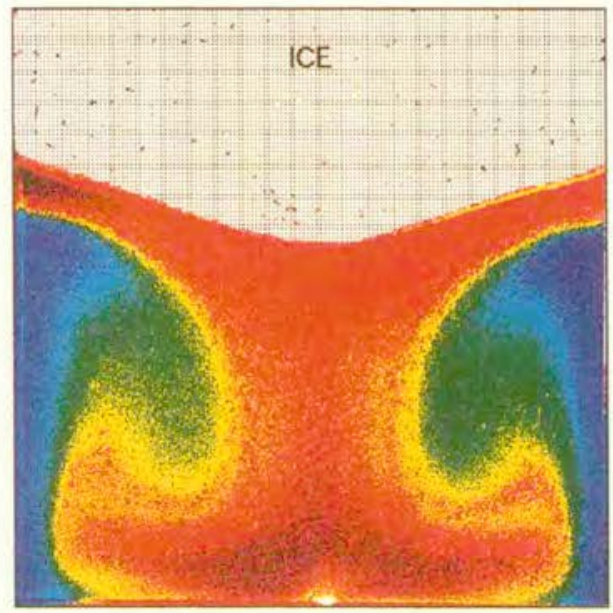

Lid cooled cavity with Plexiglas walls. Freezing of water from the top. Temperature of the top wall $T_{c}=-10^{\circ} \mathrm{C}$, temperature of the external bath $T_{h}=20^{\circ} \mathrm{C}$. Blue colour starts at $8^{\circ} \mathrm{C}$.

a)

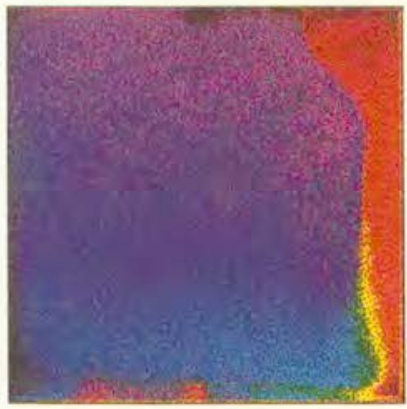

b)

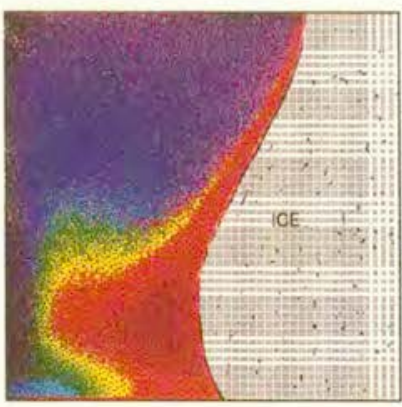

Differentially heated cavity: $T_{h}=10^{\circ} \mathrm{C}, T_{c}=-10^{\circ} \mathrm{C}$. Freezing of water from the right wall. (a) - time $=60 \mathrm{~s}$ after cooling starts. Initial supercooling visible, red plum of water at temperature below $-4^{\circ} \mathrm{C}$ penetrates the upper right corner of the cavity; (b) - time $=6000 \mathrm{~s}$, natural convection in freezing water, blue starts at $4^{\circ} \mathrm{C}$. 